Evaluación del Servicio Público de Empleo: sus efectos en la inserción laboral formal en el Área Metropolitana de Barranquilla, Colombia

Kenkin Morales-González, William Manjarrés de Ávila y Sadan de la Cruz-Almanza 
Lecturas de Economía, 91 (julio-diciembre 2019), pp. 211-239

Kenkin Morales-Gon₹ález̧, William Manjarrés de Avila y Sadan de la Cruz-Almanz̧a

Evaluación del Servicio Público de Empleo: sus efectos en la inserción laboral formal en el Área Metropolitana de Barranquilla, Colombia

Resumen: Este artículo busca medir el efecto del Servicio Público de Empleo (SPE) sobre la probabilidad de acceder a un empleo formal en el área metropolitana de Barranquilla, Colombia. Para lograr este objetivo, se combinan dos fuentes de información: los datos de personas que se registraron ante el SPE en Barranquilla y una encuesta a 400 personas registradas en el mismo servicio. Con estos datos, se estima el efecto promedio del SPE mediante la técnica de emparejamiento por puntaje de propensión (propensity score matcbing). Los resultados muestran que participar en los servicios ofrecidos por el SPE incrementa la probabilidad de conseguir empleo formal en alrededor de un $12 \%$. Este tipo de estudio es de especial utilidad para la elaboración de politicas públicas, ya que logra identificar los efectos de la intervención del Estado en la empleabilidad de una de las ciudades más importantes de Colombia.

Palabras clave: evaluación de impacto; Servicio Público de Empleo; inserción laboral; emparejamiento por puntaje de propensión.

Clasificación JEL: C15, C52, J01, J18.

Assessing the Employment Public Service: its effects on formal labour insertion in the metropolitan area of Barranquilla, Colombia

Abstract: This article seeks to measure the effect of the Employment Public Service (SPE) on the probability of accessing formal employment in the metropolitan area of Barranquilla, Colombia. To achieve this objective, two sources of information are combined: the data on people who registered with the SPE in Barranquilla, and a survey of 400 people registered with the same service. With these data, an estimate of the average effect of the SPE is obtained through propensity score matching. The results show that participating in the services offered by the SPE increases the probability of getting formal employment by around $12 \%$. This study is of special importance for the design of public policies, as it identifies the effects of state intervention in employability in one of the most important cities in Colombia.

Keywords: impact assessment; Employment Public Service; labour insertion; propensity score matcbing.

JEL Classification: C15, C52, J01, J18.

Évaluation du Service Public de l'Emploi : ses effets sur l'insertion professionnelle dans la région métropolitaine de Barranquilla, Colombie

Résumé: Cet article cherche à mesurer l'effet du Service Public de l'Emploi (PES) sur la probabilité de trouver à un emploi formel dans la région métropolitaine de Barranquilla, Colombie. Pour atteindre cet objectif, deux sources d'information sont combinées: les données des personnes inscrites auprès du SPE à Barranquilla et une enquête menée auprès de 400 personnes inscrites dans le SPE. Avec ces données, l'effet moyen de la SPE est estimé par la technique d'appariement par score de propension (propensity score matching). Les résultats montrent que la participation aux services offerts par le SPE augmente la probabilité de trouver un emploi formel d'environ $12 \%$. Ce type d'etude est particulièrement utile pour la conception de politiques publiques, car il permet d'identifier les effets de l'intervention de l'État sur l'employabilité d'une des villes les plus importantes de Colombie.

Mots clés: évaluation d'impact; Service Public d'Emploi; insertion du travail; appariement par score de propension.

Classification JEL: C15, C52, J01, J18. 


\title{
Evaluación del Servicio Público de Empleo: sus efectos en la inserción laboral formal en el Área Metropolitana de Barranquilla, Colombia*
}

\author{
Kenkin Morales-González (DiD ${ }^{\mathrm{a}}$, William Manjarrés de Ávila $\mathbb{D}^{\mathrm{b}}$ y Sadan \\ de la Cruz-Almanzac ${ }^{\mathrm{c}}$
}

-Introducción. -I. Revisión de literatura. -II. Metodología y datos. -III. Resultados.

-Conclusiones. -Anexos. -Referencias.

doi: 10.17533/udea.le.n91a07

Primera versión recibida el 30 de septiembre de 2017; versión final aprobada el 20 de marzo de 2019

\section{Introducción}

El principal objetivo de un Servicio Público de Empleo (SPE) es facilitar a través de sus múltiples componentes, el encuentro, pertinente e idóneo, entre las personas que buscan trabajo (ya sea porque son desempleadas o porque quieren cambiar de empleo) con las vacantes ofrecidas formalmente por las empresas públicas y privadas.

En Colombia, durante el año 2011, tras la escisión del Ministerio de Protección Social, se estableció la estructura del Ministerio del Trabajo, y en 2013, bajo la creación del mecanismo de protección al cesante según la Ley

* Este trabajo es resultado de un proyecto de investigación desarrollado por el ORMET Atlántico en el marco de la convocatoria y financiamiento del Programa de las Naciones Unidas para el Desarrollo (PNUD).

a Kenkin Morales-González: docente de la Universidad del Atlántico, Colombia. Dirección postal: Carrera 30 N 8-49 Puerto Colombia, Atlántico. Dirección electrónica: kenkinmorales@uniatlantico.edu.co. https://orcid.org/0000-0001-9469-985X

b William Manjarrés de Ávila: docente de la Universidad del Atlántico, Colombia. Dirección postal: Carrera $30 \mathrm{~N}^{\circ}$ 8-49 Puerto Colombia, Atlántico. Dirección electrónica: williammanjarres@mail.uniatlantico.edu.co.https://orcid.org/0000-0001-8128-4778

c Sadan de la Cruz. Almanza: investigador del Semillero de Investigación ORMET Uniatlántico, Colombia. Dirección postal: Carrera 30 N 8-49 Puerto Colombia, Atlántico. Dirección electrónica: sadandelacruz@mail.uniatlantico.edu.co. 
Morales-González, Manjarrés de Ávila y Cruz-Almanza: Evaluación del Servicio Público...

1636, se fortaleció financiera y administrativamente la Unidad Administrativa del Servicio Público de Empleo como unidad encargada de administrar el SPE y la red de prestadores del mismo.

A partir de estos cambios legales, se evidenció también la transformación del SPE, el cual pasó de un modelo basado en la intermediación laboral pura a un modelo integral de gestión y colocación de empleo que, a través de su denominada red de prestadores, ofrece múltiples servicios a empleadores y a postulantes en todos los departamentos del país.

Con el objetivo de aportar al fácil encuentro entre la oferta y la demanda laboral, una de las políticas activas que el Ministerio del Trabajo ha venido desarrollando a través de la operación del nuevo SPE son los denominados Centros de Empleo. De acuerdo con el Observatorio del Servicio Público de Empleo (2016a) estos centros son "espacios físicos que ofrecen diferentes servicios básicos de gestión y colocación de empleo como el registro de hoja de vida, orientación laboral, preselección y remisión a empresas, y en algunos casos diversos servicios complementarios como la remisión a procesos de certificación de competencias, capacitación para el trabajo, programas de emprendimiento y oferta de programas sociales en el nivel local y nacional. Por los múltiples servicios que ofrece, los centros de empleo son la principal estrategia de intervención del Servicio Público de Empleo" (2016a, p. 4).

La transformación del SPE, que cuenta con múltiples servicios de orientación para la gestión de empleos y que recibe información de los procesos de gestión del capital humano de las empresas públicas y privadas, lo ha consolidado como una estrategia de la cual se espera un aporte significativo para el cierre de las brechas que alejan al mercado del trabajo colombiano de la senda del trabajo decente.

Puesto que Colombia y Latinoamérica se enfrentan a dificultades significativas en el desempeño de su mercado laboral en general, es importante analizar y conocer los factores que inciden en el resultado positivo obtenido por territorios como Barranquilla y su área metropolitana, donde se ha logrado mantener una tasa de desempleo de un dígito y menor al promedio nacional desde el año 2011 (8,2\%), lo que la convirtió para el cuarto trimestre del año 2016 en el área metropolitana del país con menor tasa de desempleo 
(7,3\%) (datos de la Fuente de Información Laboral de Colombia -FILCOdel Ministerio del Trabajo).

Sin embargo, es importante resaltar la situación de este territorio, ya que, a pesar de su alentador contexto en el desempleo, el desempeño laboral del área metropolitana se ve afectado por otros problemas del mercado, como "un mercado del trabajo secundario, en el cual los puestos son malos, los salarios son bajos, y la rotación es alta" (Blanchard, 2000, p. 111); por tanto, estos problemas requieren una intervención articulada y activa entre el Estado y las empresas.

Uno de estos problemas es la brecha entre géneros. Para el caso del desempleo, durante los trimestres de los años 2013 a 2016, la tasa promedio para las mujeres ha sido de 5,7 puntos porcentuales mayor que la tasa masculina, y es una de las menores a nivel regional. Así mismo, según el Observatorio Regional del Mercado de Trabajo (ORMET Atlántico), en promedio, existe una brecha de 21,2 puntos porcentuales en la tasa de ocupación entre hombres y mujeres para el mismo período (ORMET, 2017).

Sumado a esta situación, se identifica una tasa de informalidad superior al $50 \%$ de la población ocupada, muy a pesar de contar con una tendencia a la baja durante los cuatro trimestres del año 2016. Para los trimestres del período 2014-2016, en promedio, la informalidad masculina fue de 59,2\%, y la femenina de 65,3\% (ORMET Atlántico, 2017).

De otro lado, el promedio de ingresos derivados del trabajo durante los años 2013-2016 fue de \$920.984 para Barranquilla y Soledad, por lo que esta área se ubicó de sexta entre las siete áreas metropolitanas y quedó solo por encima de Cúcuta, cuyo ingreso promedio para el mismo período fue de $\$$ 771.774 .

Por su parte, la tasa de desempleo juvenil (población con edades entre los 18 y 28 años) fue, en promedio, de 17,4\% durante los años 20142016, y superó la tasa a nivel nacional, que en promedio alcanzó el 15,7\% para el mismo período (datos de la Gran Encuesta Integrada de Hogares -GEIH- del DANE, 2017). Según el centro de investigación Abdul Latif Jameel Poverty Action Lab (J-PAL), el desempleo en este grupo poblacional, frente al creciente número de graduados en educación superior, se considera 
Morales-González, Manjarrés de Ávila y Cruz-Almanza: Evaluación del Servicio Público...

un indicador de desajustes y problemas de pertinencia de la educación, entre otros problemas y barreras que enfrenta esta población (J-PAL, 2017).

Según datos del Observatorio Laboral para la Educación (OLE), entre los años 2010 y 2016 en el Área Metropolitana de Barranquilla se ha duplicado el número de graduados en pregrado (formación técnica profesional, tecnológica y universitaria), pasando de 8.626 graduados en 2010 a 17.997 graduados en 2016, pero no ocurrió lo mismo con su empleabilidad.

De las políticas activas desarrolladas por el Ministerio del Trabajo, el SPE ha sido la de mayor cobertura e incluye multiplicidad de servicios. No obstante, este se ha implementado de acuerdo con el contexto de cada territorio de manera diferencial y progresiva, mediante la operación creciente de puntos de información, centros de empleo acogidos por entidades territoriales, agencias de empleo en el seno de las regionales del Servicio Nacional de Aprendizaje (SENA) y de las cajas de compensación familiar, así como la autorización de prestadores en universidades y otras instituciones privadas.

A nivel nacional, durante el período 2013 a 2015 se registraron en el SPE 1.066.853 personas, y durante el año 2016 593.430, año en el cual el $55,9 \%$ de los registrados fueron mujeres y el 44,1\% hombres. La mayor participación por nivel educativo se concentró en aquellos postulantes con educación secundaria $(30,8 \%)$ y universitaria $(16,1 \%)$.

Para el Caribe Colombiano, los datos del Observatorio del Servicio Público de Empleo (2016b) identifican a Cartagena y Barranquilla como territorios líderes en la implementación del SPE, pues durante el año 2016 Cartagena fue la ciudad con mayor número de buscadores de empleo inscritos, con 128.284, seguida de Barranquilla, con 98.487. De estos buscadores, según el máximo nivel educativo alcanzado, el 37,3\% manifestaba tener formación técnica o tecnológica, 31,9\% educación secundaria y el 25,3\% formación universitaria.

En cuanto a las vacantes registradas, se contó con 84.764 registros distribuidos en las siguientes cuatro ciudades: $38 \%$ en Cartagena, 36,5\% en Barranquilla, $14,1 \%$ en Montería y $5,6 \%$ en Sincelejo. De estas vacantes, destacan el 16,4\% que están concentradas en el sector comercio, $12,4 \%$ en 
sector de industrias manufactureras, incluyendo la fabricación de sustancias y productos químicos, y en tercer lugar el 11,8\% del sector de actividades del servicio administrativo y apoyo.

Respecto a la colocación de las personas que buscan empleo, Cartagena sigue liderando entre las cuatro ciudades del Caribe, pues durante el 2016 tuvo 12.481 colocados, seguida de Barranquilla con 16.547, Montería con 8.291 y Sincelejo con 3.193 .

De acuerdo con las problemáticas descritas y con los indicadores de gestión del SPE en el territorio, desde el ORMET Atlántico se consideró pertinente conocer de forma rigurosa el impacto de la política activa del SPE y, así mismo, identificar posibles decisiones de política en el territorio.

Para evaluar los efectos del SPE en Barranquilla y su área metropolitana, se aplicó una metodología cuasi-experimental que comparó los resultados entre un grupo de inscritos en el SPE que se postularon al menos a una vacante y recibieron alguno de los servicios de orientación (grupo de tratamiento), con otro colectivo de individuos inscritos, con características similares, pero que no recibieron alguno de los servicios de orientación (grupo de control). Los resultados verificaron que esta estrategia logró un impacto positivo en la inserción formal al mercado laboral de la población en general.

El presente artículo se divide en cuatro capítulos. En el primero, se describen los servicios de intermediación laboral y las evaluaciones de impacto de servicios para la empleabilidad; en el segundo, se explica la metodología de evaluación de impacto aplicada; en el capítulo tercero, se muestran los resultados de la evaluación y, finalmente, se exponen las conclusiones de la investigación.

\section{Revisión de literatura}

\section{A. Servicios y funcionamiento del SPE}

La dinámica del mercado laboral corresponde al espacio donde se configuran "la interacción entre la oferta laboral correspondiente a los trabajadores que ofrecen su fuerza de trabajo, y la demanda laboral 
Morales-González, Manjarrés de Ávila y Cruz-Almanza: Evaluación del Servicio Público...

correspondiente a los empresarios que requieren explotación de este factor" (Cano \& Ochoa, 2008, p. 118).

Así, de acuerdo con Prieto (2009), la competencia imperfecta que caracteriza al mercado de trabajo (dada la asimetría de información) justifica la existencia de procesos de intermediación laboral, definida como "el conjunto de acciones que tienen como objeto poner en contacto los oferentes de trabajo con los demandantes de empleo para su colocación" (Rojas, 2011, p. 187). Dicha actividad reduce los costos en la búsqueda de empleo y atenúa la selección adversa presente en este tipo de mercados (Prieto, 2009).

En este sentido, el SPE es una importante herramienta para coordinar procesos de intermediación laboral. De acuerdo con el Banco Interamericano de Desarrollo, la Asociación Mundial de los Servicios Públicos de Empleo y la Organización para la Cooperación y el Desarrollo Económico (2015), los servicios más importantes ofrecidos por los SPE son: colocación laboral (o intermediación), ejecución de políticas activas de mercado laboral y generación de información sobre el mercado de trabajo. Dado estas características, dichos servicios públicos son denominados de intermediación laboral.

Adicional a estos, se han identificado otros tipos de atención que paulatinamente se han incorporado a unidades de servicios públicos de empleo, como es el caso colombiano y de su red de operadores, a través de los cuales se brinda: asistencia en la búsqueda de empleo y colocación o intermediación laboral, capacitación, orientación a emprendedores, información sobre el mercado laboral y administración del seguro de desempleo.

El uso de estos servicios de intermediación laboral, permite una mayor formalización del mercado de trabajo (Uribe \& Ortiz, 2006; y Uribe, Viáfara \& Oviedo, 2007); así pues, dichos "canales formales"1 contribuyen al acceso

1 De acuerdo con Uribe, Viáfara y Oviedo “[...] los canales formales se basan en normas de vinculación laboral que están al acceso de todas las personas que cumplen con los requisitos establecidos; estos canales pueden ser privados, como las agencias de contratación temporal, o públicos, como los centros de información para el empleo del SENA” (2007, p. 46). Además, según ellos, dichos canales, no cuentan con barreras de entrada distintas a los requerimientos 
de empleos de mayor calidad, con mayor efectividad en relación a las necesidades de capital humano de las empresas, y a un mejor bienestar para los trabajadores.

A nivel nacional, la red de instituciones prestadoras del SPE incluye: cajas de compensación familiar, alcaldías, gobernaciones, bolsas de empleo de universidades, agremiaciones, la agencia pública de empleo a cargo del SENA, y las diferentes agencias privadas. El SPE es gratuito y cuenta con información disponible en una plataforma web que, previa entrega de licencia por parte del Ministerio del Trabajo, habilita dichas instituciones para prestar el servicio de empleo basado en cuatro componentes: gestión empresarial, registro de postulantes, orientación psicológica e intermediación laboral. Adicionalmente, en algunos puntos denominados centros de empleo, se presta el servicio de capacitación o formación para el trabajo, a través de los programas de formación disponibles en el SENA y otros programas creados con base en la demanda empresarial.

En el caso particular del Área Metropolitana de Barranquilla, el SPE opera a través de seis instituciones prestadoras (tres públicas y tres privadas), diez bolsas de empleo avaladas a Instituciones de Educación Superior del territorio, cinco centros de empleo que consolidan trece puntos de atención en toda el área metropolitana y un punto de información. Cabe aclarar que, para el análisis desarrollado a lo largo de este estudio, se tomaron en cuenta nueve agencias de empleo con presencia en los cinco municipios del Área Metropolitana: Combarranquilla, Comfamiliar, Cajacopi, Área Metropolitana de Barranquilla, los servicios de empleo de cada uno de los cuatro municipios (Malambo, Galapa, Puerto Colombia, y Soledad), y el Centro de Oportunidades de Barranquilla.

Cada una de estas instituciones presta el servicio de intermediación de acuerdo con los lineamientos del SPE. En el caso de los buscadores de empleo, la primera etapa denominada recepción es donde se identifican las necesidades del aspirante, se recolecta de manera virtual o presencial sus datos

por los puestos de trabajo, así como el costo directo, que se relaciona con los costos de clasificación, el uso de internet, entre otros. 
Morales-González, Manjarrés de Ávila y Cruz-Almanza: Evaluación del Servicio Público...

personales y laborales, y se hace el direccionamiento a los servicios pertinentes según su situación.

La segunda etapa, denominada orientación laboral, es el momento en el cual se analiza el perfil ocupacional del buscador, enfatizando en sus intereses, motivaciones, necesidades, saberes, habilidades. En el marco de esta etapa se provee información del contexto en el que se desarrollan las diferentes ocupaciones. Aquí se opera de acuerdo con dos tipos de orientación. En la orientación laboral entrevista se busca reelaborar el perfil del buscador, analizando sus capacidades y competencias, en relación con sus expectativas y la situación del mercado laboral; finalmente, se diseña conjuntamente una ruta ocupacional, que incluye actividades como capacitaciones, información sobre vacantes vigentes, programas de formación, subsidios, entre otros. En la orientación laboral talleres, a partir de una multiplicidad de técnicas lúdicas, intelectuales, individuales o grupales, se promueve una circulación horizontal de los conocimientos en torno al contexto laboral, como elaboración de sus proyectos ocupacionales, elaboración de cartas de presentación y otras herramientas para optimizar la búsqueda de empleo, entre otros.

Por el lado de la demanda laboral, se cuenta con una etapa denominada gestión empresarial, en se realiza de forma gratuita la inscripción y orientación a empleadores para el debido registro de sus vacantes en la plataforma. Adicionalmente, se brinda información básica sobre legislación laboral, programas de empleo, incentivos para la contratación de personal e inducción para la utilización de la plataforma SISE, lo que promueve el trabajo digno, decente y no discriminatorio. Durante esta etapa de acompañamiento a las empresas en todo el proceso de gestión de capital humano, el empleador puede acceder a otro tipo de servicios, como capacitación a la medida, pruebas psicotécnicas, pruebas performance, entre otros.

Finalmente, en la etapa de gestión de vacantes o intermediación laboral, ofrecida por cada uno de los prestadores, se brinda una asesoría en la definición y publicación de la vacante; luego, se lleva a cabo un proceso de emparejamiento entre la vacante y la oferta de buscadores. Una vez identificados potenciales empleados, se realizan procesos previos de verificación de información de los oferentes y entrevistas de preselección. 
Seguidamente, se remiten a las empresas los perfiles y se da seguimiento a cada uno.

\section{B. Evaluaciones de impacto de servicios de intermediación laboral}

Las evaluaciones de impacto de programas de intermediación laboral (incluyendo la evaluación específica de programas y servicios ofrecidos por unidades integrales de intermediación laboral) más relevantes en la literatura se concentran en el impacto directo de los participantes, y son pocas las que dedican especial atención a los efectos colaterales. Así mismo, se encuentran investigaciones relacionadas con las unidades de SPE de distintos países y algunas evaluaciones puntuales sobre servicios específicos.

En este sentido, para el caso chileno, Chanamé $(1999)^{2}$ muestra que las oficinas municipales de información laboral tienen una eficiencia dos a uno; es decir, por cada dos oportunidades de empleo, el sistema solo tiene la capacidad para realizar una colocación.

Por su parte, Vera (2005) aplicó para el contexto peruano una estimación de emparejamiento por puntaje de propensión (PSM) ${ }^{3}$ y de duración con regresión simple, de la cual concluye que los hombres desempleados cesantes con altos niveles de educación tienen una reducción en la duración del desempleo. Sin embargo, tener más años de edad aumenta significativamente esta variable.

De los casos identificados en México, encontramos que Flores (2010) realiza una evaluación sobre el servicio de intermediación mexicano a través de la aplicación de un modelo probit. De sus resultados resalta que entre los beneficios de las personas que acceden a estos servicios está el recibir el doble de ingresos vía salarios, en contraste con las personas que encuentran empleo con otros canales e, igualmente, la reducción de menos del doble de las horas trabajadas por semana. Por su parte, Van Gameren (2010) sostiene que la participación en los programas Empleo Formal y Bécate incrementan

2 Si bien la investigación de Chanamé utiliza metodologías no relacionadas con evaluación de impacto, se considera pertinente para la temática general abordada en el estudio.

3 Sigla del término en inglés Propensity Score Matching. 
Morales-González, Manjarrés de Ávila y Cruz-Almanza: Evaluación del Servicio Público...

los ingresos salariales de los mexicanos, en el primer caso, con un aumento de 1.752 pesos mensuales y, en el segundo programa, con un incremento de 1.075 pesos por mes. Otro aporte importante de la utilización de los servicios de intermediación es la mejora en la tasa de colocación del mercado, la cual se ubica entre 42 y 53 puntos porcentuales para los participantes de estos programas.

Por otro lado, Castillo, Ohaco y Schleser (2015) aplican modelos de PSM y probit para la evaluación de impacto de la política argentina de formación profesional sectorial (los mismos modelos utilizados en la presente investigación). Los autores concluyen que la participación en estos servicios aumenta la probabilidad de inserción laboral en 3 puntos porcentuales, en comparación con los no beneficiados, y aumenta la inserción laboral en un $21 \%$. Así mismo, encuentran que la edad es la variable más significativa relacionada con la empleabilidad formal, dado que la probabilidad de acceder a un empleo aumenta a medida que se reduce la edad de las personas, es decir que los jóvenes son quienes tienen mayor probabilidad de insertarse en el mercado de trabajo.

En Colombia, a nivel de país, se han realizado evaluaciones a programas de empleo como: Empleo en Acción, orientado a brindar ocupación transitoria a mano de obra no calificada de los niveles 1 y 2 del Sisbén ${ }^{4}$. En esta evaluación, el impacto en el desempleo fue medido según la posición ocupacional, la cual mejoró en $2 \%$ a los asalariados, $0,5 \%$ en independientes; así mismo, se registró una disminución de 3,6 \% en el desempleo y de 1,1\% en los inactivos (Departamento Nacional de Planeación, 2007).

De las evaluaciones en Colombia, la realizada al programa Misión Bogotá ${ }^{5}$, se aplicó la metodología de emparejamiento, pero no se identificó impacto alguno en la probabilidad de empleo de los jóvenes, ni en el ingreso percibido (Alcaldía Mayor de Bogotá, 2014).

4 Sistema de identificación de Potenciales Beneficiarios de Programas Sociales que utiliza el gobierno colombiano para focalizar el gasto público. Los niveles 1 y 2 corresponden a la población más vulnerable.

5 Fue un programa de vinculación de jóvenes como guías cívicos, en el marco del cual recibieron algún tipo de formación para el trabajo. 
Por su parte, el programa denominado Jóvenes en Acción, que funciona en todo el país, evidenció impactos positivos en la probabilidad de trabajar de las mujeres (entre 10 y $12 \%$ ) frente a los resultados sin significancia estadística para los hombres. Así mismo, se registró un significativo aumento de $40 \%$ en la probabilidad de conseguir trabajo con contrato escrito y en el sector formal, y un aumento de ingresos, tanto para personas asalariadas como autónomas (Departamento Nacional de Planeación, 2008).

La evaluación de impacto del programa Jóvenes Rurales Emprendedores del SENA, realizada en el año 2009, evidenció un aumento de la probabilidad de empleo de entre un $13 \%$ y $14 \%$, un aumento de la satisfacción con el empleo y un cambio positivo en los ingresos de los beneficiarios (Fedesarrollo, 2009).

En cuanto a la evaluación del entrenamiento a buscadores, como uno de los componentes de los servicios públicos de intermediación, el J-PAL consolida resultados de evaluación de programas para la empleabilidad a población joven, basados principalmente en entrenamiento en habilidades blandas y habilidades técnicas, y en la combinación de estos con entrenamientos al interior de las empresas. Los principales hallazgos muestran resultados positivos en América Latina, en programas que combinan entrenamiento en salones de clases y algún tipo de entrenamiento al interior de empresas. De otra parte, En Estados Unidos, los resultados más exitosos se evidencian en entrenamientos que se realizan en las empresas. Para ambas regiones la relación es positiva con la empleabilidad, la mejora de ingresos, entre otras variables. En casos específicos como el programa de Colombia Jóvenes en Acción, los resultados se mantienen en el largo plazo.

No obstante, se manifiesta la necesidad de diferenciar el impacto según cada tipo de entrenamiento o programa. Esta recomendación es sustentada por Heckman, Lalonde y Smith (1999), quienes argumentan que, debido a la alta descentralización de este tipo de intervención de entrenamientos en salones de clase, es posible encontrar diferencias sustanciales en el impacto entre individuos y entre programas.

En síntesis, existe suficiente y rigurosa evidencia de los impactos generados por los servicios de intermediación laboral, y por los programas 
de formación para el trabajo. En el contexto internacional, las investigaciones revelan la importancia de evaluar con metodologías rigurosas la relevancia de los servicios de intermediación laboral, para conocer los impactos que estos tienen en sus beneficiarios. Entre las investigaciones, se destacan los análisis en términos de probabilidad de colocación identificadas en Chile y México, el impacto en el aumento de ingresos en México para el programa Becáte, entre otros, y la disminución de la duración del desempleo evidenciada para el caso de los hombres cesantes en Perú.

Para el caso colombiano, los resultados más comunes en las evaluaciones disponibles son: el impacto en la probabilidad de empleo, el aumento de los ingresos, como en el caso de Jóvenes Rurales del SENA, y el aporte a la formalización laboral del programa Jóvenes en Acción (JeA). Este último programa destaca por su aporte al cierre de la brecha de empleo entre hombres y mujeres de este grupo poblacional. Con base en estos resultados, la elección de la metodología a aplicar en el caso de Barranquilla y su área metropolitana consideró el contexto, la información disponible y la forma de implementación del SPE en este territorio.

\section{Metodología y datos}

\section{A. Estimación del impacto a través de PSM}

De los métodos cuantitativos de análisis, la evaluación de impacto provee una de las formas para determinar la causalidad entre dos o más variables estudiadas. Para su aplicación, se requiere la construcción y el análisis de un escenario contrafactual que permite aproximar lo que le hubiera pasado a los beneficiarios de una intervención o programa si no hubieran recibido el beneficio; lo que a su vez permite hallar la diferencia entre las variables de resultado aisladas del efecto de otros factores que pudieran haber causado cambios en los beneficiarios (Bernal \& Peña, 2014, p. 17).

Para estimar el impacto del SPE se aplicó un modelo cuasi-experimental, a través del cual se efectuaron mediciones para los dos grupos de individuos: uno beneficiado por los servicios del SPE (tratados) y otro de características semejantes, pero que no recibió el beneficio (no tratados), este último es 
también denominado como el grupo de control. El empleo de modelos cuasiexperimentales supone algunos problemas, el principal es la imposibilidad de controlar el sesgo de selección entre los dos grupos: los tratados y no tratados. No obstante, existen algunos procedimientos que permiten minimizar este riesgo.

En el presente estudio se utiliza la metodología PSM, con la cual se compara el grupo de tratamiento (aquellas personas que se inscribieron en el SPE, se postularon a vacantes disponibles por lo menos en una ocasión y, además, recibieron al menos un servicio de orientación) con el grupo de control (grupo de personas que también se inscribieron en el SPE pero que no se postularon a ninguna vacante laboral, ni recibieron algún tipo de servicio). La inscripción en la plataforma del SPE actúa como un elemento homogeneizador y evidencia la existencia de una búsqueda activa de empleo.

En términos generales, esta metodología se basa en el supuesto de independencia condicional estándar, donde, dependiendo de $\mathrm{X}$, la variable de tratamiento es independiente de los resultados potenciales (Becker \& Ichino, 2002). El PSM calcula el efecto medio del tratamiento sobre el grupo de individuos que participan en el programa (ATT), que se define como la diferencia en el resultado promedio de la variable de impacto (probabilidad de inserción laboral) entre aquellos que fueron tratados $(T=1)$ (los que participaron en los servicios ofrecidos por SPE) y los que no fueron tratados $(T=0)$ en la forma de:

$$
A T T=E\left[P r_{\text {Inserción }} \mid T=1, b(X)\right]-E\left[P r_{\text {Inserción }} \mid T=0, b(X)\right],
$$

donde $E[*]$ denota la operación de esperanza matemática, $T$ es la variable de tratamiento (haber participado en alguno de los servicios ofrecidos por SPE) y $b(X)$ es la puntuación balanceada derivada de las covariables observadas en $X$, de manera que la distribución condicional de $X$, dado $b(X)$, sea la misma para los grupos de tratamiento y control.

De acuerdo con el supuesto de independencia condicional estándar en el PSM, la participación está basada únicamente en características observables de los individuos; de lo contrario, los resultados obtenidos en la evaluación estarían sesgados. En este caso, el sesgo sería el resultado de la potencial correlación entre las variables no observadas ( $\mathrm{y}$, por tanto, no medidas) que 
afectan la decisión del individuo de acceder a los servicios del SPE y la variable de resultado de la evaluación, en este caso, de la probabilidad de inserción de las personas en el empleo asalariado.

Por otro lado, la aplicación adecuada del PSM requiere del uso de información de corte transversal para ambos grupos, que incluya las características observables relevantes de la decisión de participación en los servicios del SPE (las cuales deben ser variables de pretratamiento, con el fin de garantizar que no han sido afectadas por este) y las variables de resultado relevantes. Para lo anterior, se dispone de información sobre las personas inscritas en el portal web del SPE o que concurrieron a sus oficinas operadoras y registraron su información durante el año 2014. Como parte de esta información registrada se incluyen, en calidad de variables de control, características observables identificadas a partir de la teoría de capital humano en relación con la dinámica de los mercados de trabajo, para la cual Becker (1964) y Mincer (1974), consideran a la educación y la experiencia como elementos esenciales para el rendimiento de este tipo de capital.

A partir de las encuestas realizadas a la muestra seleccionada, se pudo conocer si la persona cuenta con experiencia laboral formal previa, así como la variable de resultado de la evaluación, que está representada por la probabilidad de inserción de las personas en el empleo asalariado a finales de 2016. Entonces, el impacto neto atribuible al SPE será el resultado de la diferencia entre la probabilidad de inserción laboral a un empleo formal observada entre el grupo tratamiento y de control.

\section{B. Datos}

La principal fuente de información corresponde a los datos brindados por el Observatorio del Servicio Público de Empleo a nivel nacional, quien compila la información de los territorios y brinda acompañamiento a las oficinas a nivel regional. Otros datos fueron recolectados a través de una encuesta aplicada durante diciembre de 2016, cuyo cuestionario se muestra en el Anexo, y la cual se realizó como un seguimiento, con el objetivo de contar con variables que permitieran estimar la probabilidad de inserción laboral formal de estas personas para el año 2016. 
La muestra corresponde a 400 individuos de la población total inscrita en la base de datos del SPE, escogidos de manera aleatoria y estratificada. Su método de selección garantiza un nivel mínimo del 95\% de confianza, un error relativo no mayor al $5 \%$ y permite estimar los estratos deseados, es decir, las ciudades o municipios que conforman el área metropolitana: Barranquilla, Galapa, Malambo, Puerto Colombia y Soledad.

De los 400 oferentes a los cuales se les aplicó la encuesta de seguimiento a su vinculación laboral, el $51,5 \%$ son hombres y el restante $48,5 \%$ corresponde a mujeres, con una distribución por sexo y grupos etarios equilibrada para las edades entre 18-28 años y 29-39 años; el rango con edad mayor o igual a 40 años cuenta con una representación masculina de aproximadamente $58 \%$.

Del total encuestado, el 22,5\% afirma haber encontrado algún empleo a través de la inscripción en la plataforma del SPE; de estos, el 67,3\% con una vinculación de tipo contractual asalariado, seguido por el 27,8\% con contratación independiente. Los niveles de formación educativa con mayor vinculación son universitaria, técnica y secundaria.

\section{Análisis descriptivo de las variables}

La Tabla 1 presenta las estadísticas descriptivas de las variables recogidas como parte de este estudio. Se puede observar que la edad promedio de las personas pertenecientes a la muestra es de 30 años, y su experiencia laboral promedio es de 8 años. En cuanto al nivel educativo, la mayoría de las personas de la muestra poseen una educación entre técnica y tecnológica.

Por otro lado, sobre el uso de la plataforma web del SPE, a la pregunta: "¿Cuál es su percepción del proceso de inscripción en la plataforma del SPE y de los servicios prestados?", los encuestados contestaron que les parece difícil su uso. Finalmente, otros aspectos socioeconómicos permiten evidenciar que en la muestra hay una repartición igualitaria en la variable sexo, pues el 51,5\% de la muestra son hombres y el resto mujeres. Por otro lado, las personas tienen, en promedio, 4,5 años de experiencia laboral y el $53 \%$ de las que respondieron el cuestionario tienen al menos una persona a cargo. 
Morales-González, Manjarrés de Ávila y Cruz-Almanza: Evaluación del Servicio Público...

Tabla 1. Estadisticas descriptivas

\begin{tabular}{|c|c|c|c|c|c|c|}
\hline \multirow[b]{2}{*}{ Variables } & \multicolumn{2}{|c|}{ Grupo Tratamiento } & \multicolumn{2}{|c|}{ Grupo Control } & \multicolumn{2}{|c|}{ Total } \\
\hline & Media & $\begin{array}{l}\text { Desv. } \\
\text { Estándar }\end{array}$ & Media & $\begin{array}{c}\text { Desv. } \\
\text { Estándar }\end{array}$ & Media & $\begin{array}{l}\text { Desv. } \\
\text { Estándar }\end{array}$ \\
\hline Edad & 28,1 & 8,89 & 31 & 9,74 & 30,25 & 9,6 \\
\hline Experiencia laboral en años & 4,06 & 6,39 & 4,66 & 6,14 & 4,5 & 6,2 \\
\hline Experiencia laboral en años ${ }^{2 *}$ & 57,1 & 227,15 & 59,34 & 139,18 & 58,76 & 166,26 \\
\hline Nivel de estudio (niveles) & 3 & 0,97 & 3 & 1,22 & 3 & 1,19 \\
\hline $\begin{array}{l}\text { Percepción sobre la plataforma } \\
\text { web del SPE }\end{array}$ & 2,13 & 0,62 & 2,35 & 0,78 & 2,3 & 0,74 \\
\hline Sexo & 0,49 & 0,5 & 0,53 & 0,5 & 0,52 & 0,5 \\
\hline Tiene personas a cargo & 0,62 & 0,49 & 0,5 & 0,5 & 0,53 & 0,5 \\
\hline Total observaciones & \multicolumn{2}{|c|}{104} & \multicolumn{2}{|c|}{296} & \multicolumn{2}{|c|}{400} \\
\hline
\end{tabular}

Nota: *elevada al cuadrado.

Fuente: cálculos de los autores.

\section{Resultados}

\section{A. Resultados del PSM}

Para la implementación del PSM fue necesario limitar la cantidad de individuos de tratamiento, tanto beneficiarios como no beneficiarios del SPE, bajo la condición de no haber participado en ninguna política de empleo ni haber participado de cursos de formación profesional durante otro año posterior a 2014 .

La Tabla 2 muestra la estimación de la probabilidad de participación, utilizando tanto el grupo de tratamiento como el grupo de control. Esto se realiza a través de un modelo probit, con el fin de posteriormente estimar la probabilidad de participación predicha para cada individuo de la muestra, controlando por características observadas (Bernal \& Peña, 2014). Se puede observar que, en su mayoría, las variables seleccionadas son significativas y poseen un signo adecuado para explicar la probabilidad de participar en los servicios ofrecidos por el SPE. 
Tabla 2. Estimación de la probabilidad de participación en el SPE

\begin{tabular}{lcc}
\hline Variables & $\begin{array}{c}\text { Participación } \\
(\boldsymbol{D}=\mathbf{1} \text { participa })\end{array}$ & Error estándar \\
\hline Edad & $-0,0058018^{* *}$ & 0,0032914 \\
Experiencia laboral & $-0,0054581$ & 0,0090987 \\
Experiencia laboral al cuadrado & $0,0005167^{* *}$ & 0,0003028 \\
Nivel de estudio & $0,0918282^{* * *}$ & 0,0189508 \\
Percepción de la plataforma web del SPE & $-0,0914118^{* * *}$ & 0,0343017 \\
Sexo & $-0,043318$ & 0,044246 \\
Número de personas a cargo & 0,057225 & 0,0441202 \\
\hline Observaciones & 400 & \\
logverosimilitud & $-206,73326$ & \\
Pseudo - $R^{2}$ & 0,0981 & \\
\hline
\end{tabular}

Nota: ${ }^{*} \mathrm{p}<0,10,{ }^{* *} \mathrm{p}<0,05,{ }^{* * *} \mathrm{p}<0,01$.

Fuente: cálculos de los autores.

En el caso específico del nivel educativo, se reconoce la gran participación de personas con formación técnica y tecnológica con una tasa creciente de participación en el SPE, cuya tasa de graduados se duplicó entre los años 2010 y 2016 (datos del Observatorio Laboral para la Educación); en cambio, los registrados en el SPE con formación universitaria y de postgrado tuvieron menor representación y fueron superados en el registro por la población con educación media.

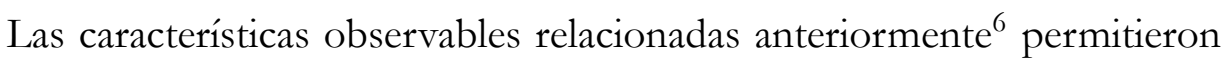
balancear la muestra y contar con dos grupos con características muy similares (en términos del propensity score estimado), donde la única diferencia observable entre ambos grupos es el estatus de grupo de tratamiento o de

6 En la presente investigación no se incluyó la variable experiencia laboral sin elevar al cuadrado (en niveles), según lo sugerido por el modelo de Mincer (1974), porque los cálculos muestran que no resultó estadísticamente significativa para el modelo. Esta razón fue fundamental para excluir otras variables que, aunque fueron recogidas por la encuesta, su significancia estaba muy alejadas de los intervalos aceptados. 
Morales-González, Manjarrés de Ávila y Cruz-Almanza: Evaluación del Servicio Público...

grupo de control. Con ellos se construyó la región de soporte común (Figura 1), donde se encuentran las probabilidades de PSM estimadas para ambos grupos. Ambas probabilidades son positivas y se entiende como la zona en donde se realizará la evaluación.

Figura 1. Región de soporte común

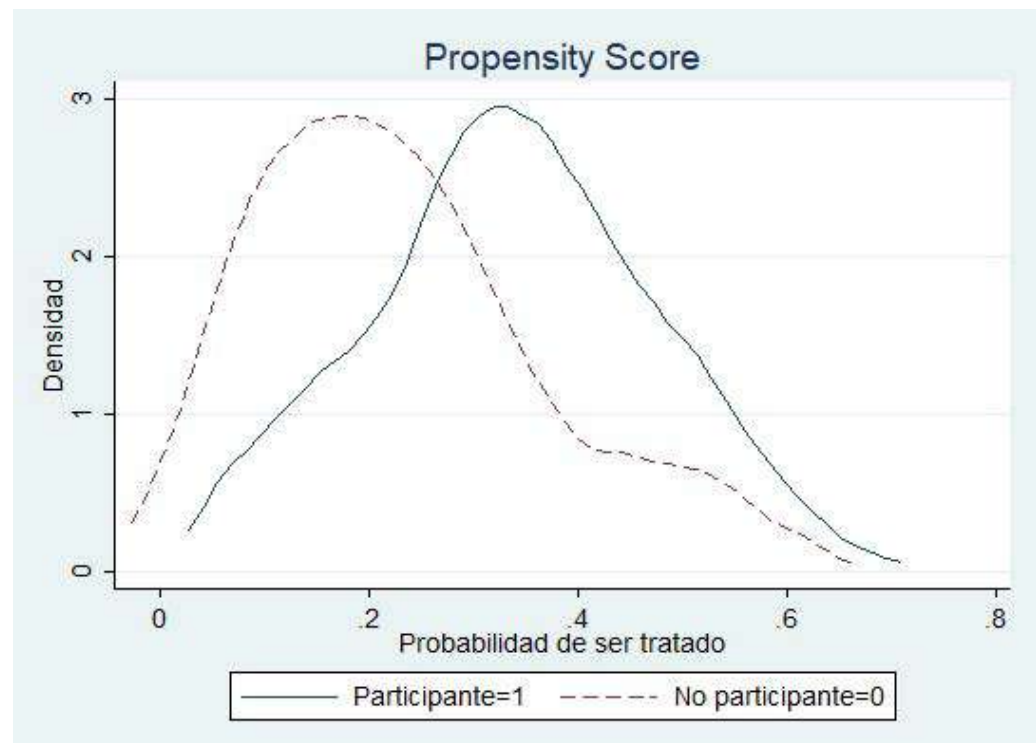

Fuente: cálculos de los autores.

A partir de la región de soporte común, se estimó el PSM, teniendo en cuenta cada uno de los algoritmos de emparejamiento: Kernel, radio de emparejamiento y vecino más cercano. Lo anterior, con el fin de contrastar los resultados observando si el efecto estimado no depende en gran medida del algoritmo utilizado. Es posible analizar la robustez de los resultados estimando el impacto por medio de múltiples algoritmos de emparejamiento (Bernal \& Peña, 2014). Adicionalmente, se verificó la calidad del emparejamiento con el fin de comprobar que exista un balance en las características observadas para ambos grupos: el de tratamiento y el de control. Por último, se estimó el efecto promedio del programa, como la 
diferencia entre la proporción de individuos con probabilidades de inserción laboral del grupo tratamiento y la del grupo de control.

\section{B. Resultados del impacto del SPE}

En la siguiente tabla se observa que de los 400 individuos del total de la muestra; 104 pertenecen al grupo de tratamiento y 296 al grupo de control. Dado el cumplimiento de las condiciones anteriores: la restricción de la muestra al soporte común, el balance de las características observadas para ambos grupos, y los algoritmos de emparejamiento, es posible evidenciar un impacto positivo en las personas que se postularon en las vacantes y accedieron a los servicios ofrecidos por el SPE, sobre su probabilidad de inserción laboral formal, dadas las condiciones de apoyo brindadas por la plataforma y las oficinas operadoras en Barranquilla y su área metropolitana.

De manera específica, se puede decir que haber participado en los servicios ofrecidos por el SPE de Barranquilla y su área metropolitana, o haber recibido algún direccionamiento como orientación laboral, incrementa la probabilidad de conseguir empleo formal alrededor de $12 \%$. Este es un impacto positivo y sólido, ya que es significativo en los tres algoritmos de emparejamiento. Además, es importante mencionar que, para cada impacto obtenido se calcularon los errores estándar, los intervalos de confianza y un test de significancia a través de la técnica estadística de bootstrap, dado que la metodología de PSM es no-paramétrica.

Por otro lado, se realizó la evaluación de impacto sobre la probabilidad de conseguir empleo formal, dividiendo la muestra en diferentes grupos de edades, entre los rangos de 18-28 años, 29-39 años y mayor o igual a 40 años. Los resultados evidencian que no existe diferencia significativa entre los rangos de edades de los individuos de la muestra que participaron en los servicios ofrecidos por el SPE de Barranquilla y su área metropolitana y recibieron algún tipo de direccionamiento.

Finalmente, con el ánimo de diferenciar los impactos de acuerdo con la formación académica de los postulantes, se dividió la muestra en niveles educativos: educación secundaria, educación técnica y tecnológica, educación 
Morales-González, Manjarrés de Ávila y Cruz-Almanza: Evaluación del Servicio Público...

en pregrado y postgrado. Al igual que en el ejercicio por grupo de edades, los resultados muestran que no existe diferencia significativa sobre la probabilidad de inserción laboral formal de los individuos en los distintos niveles. Dicho de otra forma, no existe evidencia suficiente para rechazar la hipótesis de no significancia estadística para los estimadores calculados en cada nivel educativo.

Tabla 3. Estimación del impacto del SPE a través del soporte común

\begin{tabular}{|c|c|c|c|}
\hline \multirow{2}{*}{ Muestra total } & \multicolumn{3}{|c|}{ Algoritmo de emparejamiento PSM } \\
\hline & Kernel & Radio & Vecino más cercano \\
\hline Tratamiento $(\mathrm{T})$ & 0,5339806 & 0,5339806 & 0,5339806 \\
\hline Control (C) & 0,4073798 & 0,4083874 & 0,3721683 \\
\hline Diferencia (T-C) & 0,1266008 & 0,1255932 & 0,1618123 \\
\hline \multicolumn{4}{|l|}{ Bootstrap } \\
\hline Impacto (ATT) & $0,1266008^{* *}$ & $0,1255932^{* *}$ & $0,1618123^{* *}$ \\
\hline Error estándar & 0,0613792 & 0,0605968 & 0,0818621 \\
\hline \multicolumn{4}{|c|}{ Número de observaciones en el soporte común: } \\
\hline Tratados & & 104 & \\
\hline Número de tratados & & 296 & \\
\hline Observaciones totales & & 400 & \\
\hline
\end{tabular}

Nota: ${ }^{*} \mathrm{p}<0,10,{ }^{* *} \mathrm{p}<0,05,{ }^{* * *} \mathrm{p}<0,01$.

Fuente: cálculos de los autores.

\section{Conclusiones}

En este estudio se realizaron estimaciones a través de la metodología PSM, con el fin de evidenciar los impactos que tiene la participación en servicios ofrecidos por el SPE sobre la probabilidad de conseguir empleo formal en los beneficiaros de Barranquilla y su área metropolitana. Este tipo de estudio se considera de especial utilidad en el diseño y formulación de políticas públicas, ya que, con base en argumentos rigurosos y objetivos logran identificar, de manera aislada, los efectos de la intervención del Estado. 
A partir de los resultados, se concluye que el haber participado de los servicios ofrecidos por el SPE, haberse postulado mínimo una vez en una vacante ofrecida y haber recibido algún direccionamiento como orientación laboral, aumenta la probabilidad de conseguir empleo formal o de insertarse en la vida laboral formal. Los resultados evidencian un impacto sólido en niveles de significancia; por tanto, es necesario que los gobiernos atiendan sin interrupciones iniciativas de este tipo, ya que contribuyen a mejorar la empleabilidad y aportan a disminuir las altas tasas de informalidad y brechas en el empleo a nivel local, regional y nacional.

El impacto obtenido en la probabilidad de colocación del 12,6\% de los participantes o beneficiarios del SPE en el Área Metropolitana de Barranquilla está acorde con los resultados encontrados por evaluaciones de programas relacionados que se realizaron en otros territorios. No obstante, se considera viable diseñar e implementar estrategias de formación pertinentes en los niveles de formación técnica y tecnológica, según las necesidades del contexto, además de estrategias de seguimiento a nuevas unidades de emprendimiento que aporten a la generación de vacantes formales en el territorio.

Así mismo, se sugiere potenciar la articulación de las necesidades del mercado con los programas de formación superior, sobre todo en lo relacionado con la formación universitaria, para la cual se identificó el impacto más bajo y sin significancia estadística. Esto puede ser un aspecto estratégico a la hora de ofrecer programas de formación y entrenamiento que consideren las necesidades futuras de capital humano de acuerdo con las tendencias de cada sector o rama de actividad económica.

Finalmente, se reconocen algunas limitaciones empíricas de este trabajo. Una de estas limitaciones fue que a las personas encuestadas solo se les preguntó acerca de las variables de empleabilidad de los últimos meses y, por lo tanto, no fue posible probar si los efectos del SPE sobre la inserción laboral son constantes o transitorios; sin embargo, esto podría solucionarse con el uso de datos de panel. Esta y otras limitaciones podrían ser abordadas en estudios posteriores que proporcionen mayor solidez a través del uso de datos de panel, experimentos aleatorios o variables instrumentales. 
Morales-González, Manjarrés de Ávila y Cruz-Almanza: Evaluación del Servicio Público...

\section{Anexo}

Figura 1A. Cuestionario de la encuesta aplicada
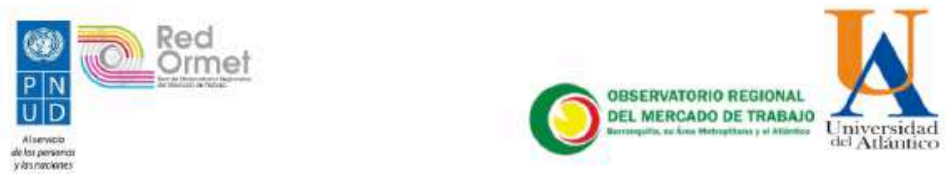

Encuesta para la evaluación de impacto del Servicio Público de Empleo en el Departamento del Attántico

Proyecto que se ejecuta en el marco del contrato entre la Universidad del Atlántico como secretaría técnica del ORMET Barrançuilla y su AM, y el Programa de la Naciones Unidas para el Desarrollo - PNUD-

\section{Datos generales di encuestado}

1. Nombre del encuestado:

2. Género: $F($ M

3. Edad if $n$ añes complinkes:

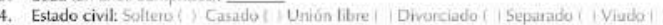

5. Personas a cargo: Si t Cuartos

6. Ciudad o municipio de residenci

- Nacionalidad 1:

8. A la fecha se encuentra en situación de discapacidad? Si I I No is

9. iA la fecha se encuentra registrado en alguna Unidad de Atención a Víctimas del Conflicto Armado? Si i i No I !

II. Educación, experiencia y otras habilidades para el trabajo.

10. Marque con una " $\mathrm{X}$ " el último nivel educativo adquirido Para el cual tenga st respectivo rftulo:

Basica primaria

Básica secundaria

Educación Media

Técnica

Tecrralógica

Profesional

Esoccialización

Maestra

Dectirat
11. In la actualidad cuenta con manejo de herramientas ofimáticas? Si i i Na if ? (Hertarnientas que permitan idear, crear, manipular, inansmitir o almarear. informaciön necresuris en una oficins - Internet. computadores Mirroscin Oificet

12. A la fecha cuenta con ticencia de conducción de vehículo o motocicleta? 5 i Nol

En caso de responder Si a la pregunta 12, licencia en la plataforma del SPE? SF t A A

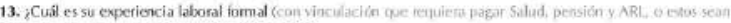

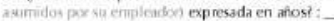

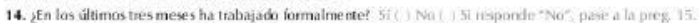

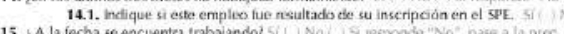

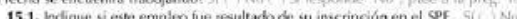

16. Jusked ha logrado conseguir algùn empleo a traves de su inscripción en la piataforna del Servicio Público de Empleo y demás servidios prestados desde su inscripción? sit i Nón

A partir de aqui responder las preguntas 16.1 a ba 16.10 preierblemerte para las personas que hay an conseguido

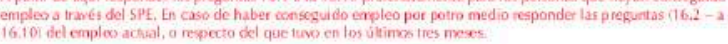




\section{Figura 1A. Continuación}

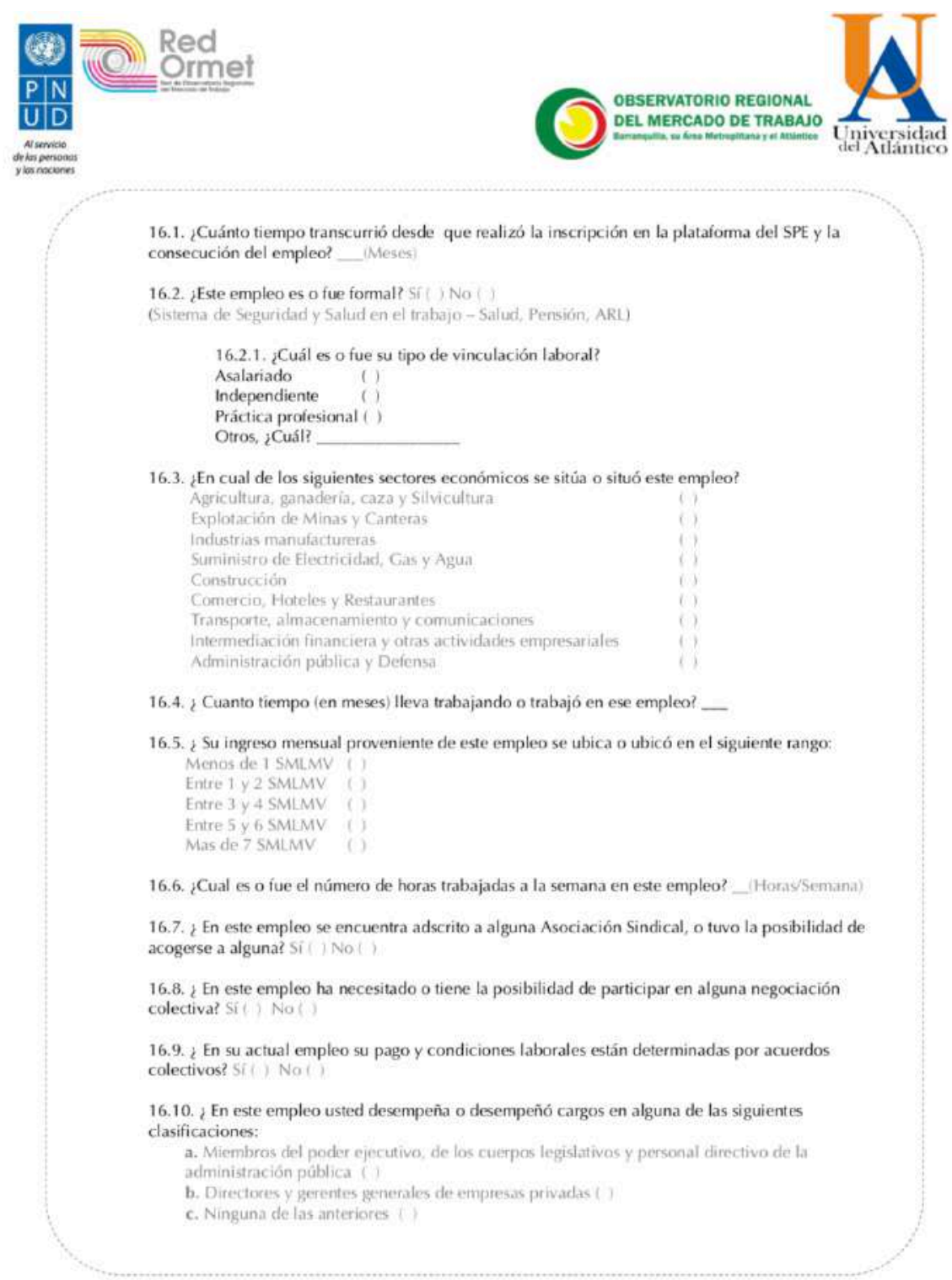

Lecturas de Economía -Lect. Econ. - No. 91. Medellín, julio-diciembre 2019 
Morales-González, Manjarrés de Ávila y Cruz-Almanza: Evaluación del Servicio Público...

Figura 1A. Continuación
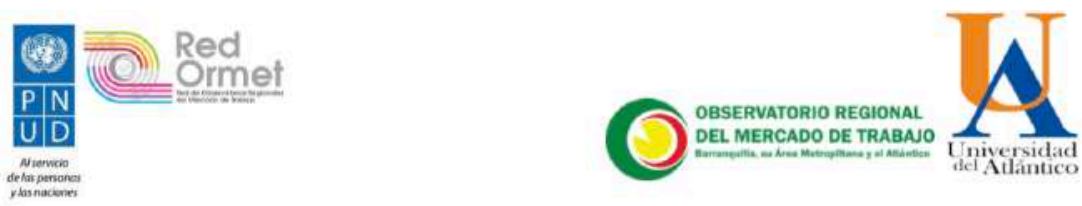

III. Percepción de la plataforma del SPE

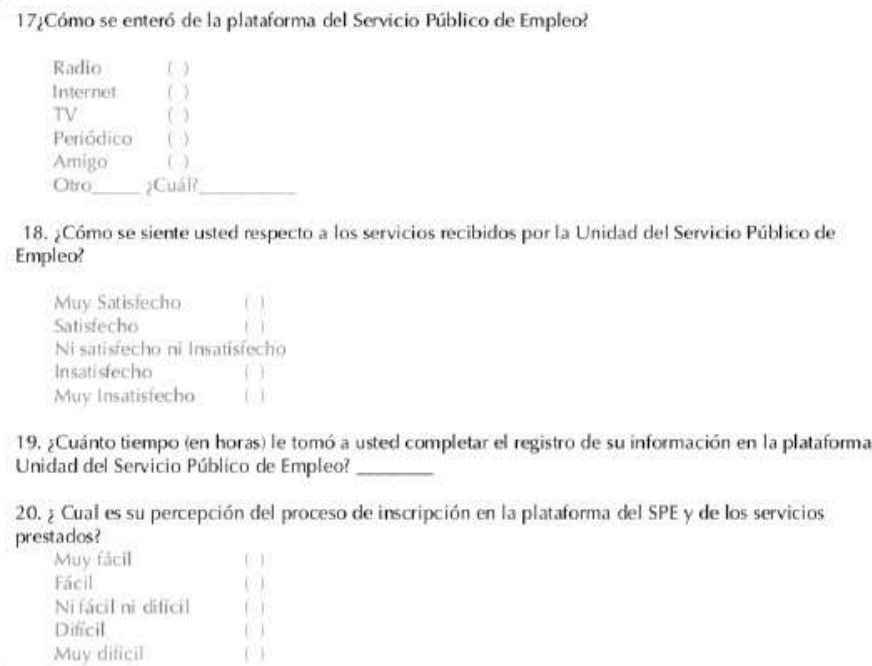

GRACIAS POR SU TIEMPO

\section{Referencias}

Abdul Latif Jameel Poverty Action Lab (J-PAL) (2017). Skills training programs. Recuperado de: https://www.povertyactionlab.org/sites/default/ files/documents/skills-for-youth-review-paper.pdf

Alcaldía Mayor de Bogotá (2014). Bogotá: ciudad de estadisticas. Evalaución de impacto del Programa Misión Bogotá. Recuperado de: http://www.sdp.gov.co/sites/default/files/evaluacion_de_impacto_del_ programa_mision_bogota.pdf 
Becker, G. S. (1964). Human Capital. New York: National Bureau of Economic Research.

Becker, S. O. \& Ichino, A. (2002). Estimation of average treatment effects based on propensity scores. The Stata Journal, 2(4), 358-377.

Bernal, R. \& Peña, X. (2014). Guía práctica para la evaluación de impacto. Bogotá D.C.: Universidad de los Andes.

Banco Interamericano de Desarrollo, Asociación Mundial de los Servicios Públicos de Empleo \& Organización para la Cooperación y el Desarrollo Económico (2015). El mundo de los servicios públicos de empleo. Desafios, capacidades y perspectivas para los servicios públicos de empleo en el nuevo mercado laboral. Recuperado de: https:/www.skillsforemployment.org/KSP/es/Details/index.htm?dn= WCMSTEST4_171856

Blanchard, O. (2000). Macroeconomía. Madrid: Prentice Hall.

Cano, C. A. \& Ochoa, A. M. (2008). Empleo, desempleo y salario real: análisis del mercado laboral de la ciudad de Medellín (1995-2006). Lecturas de Economia, 69, 117-139.

Castillo, V., Ohaco, M. \& Schleser, D. (2014). Evaluación de impacto en la inserción laboral de los beneficiarios de los cursos sectoriales de formación profesional (Serie Domumentos de Trabajo No. 6). Recuperado del sitio web de la Organización Internacional del Trabajo: https:/www.ilo.org/wcmsp5/groups/public/—americas/-rolima/_ilo-buenos_aires/documents/publication/wcms_305990.pdf

Chanamé Zapata, C. (1999). El sistema de intermediación laboral y los servicios públicos de empleo en Chile: Diagnostico, evaluación y propuesta para mejorar su gestión. Recuperado de: http://www.sistemaspublicos.cl/wpcontent/uploads/2017/04/CASO40.pdf

Departamento Administrativo Nacional de Estadísticas (DANE) (2017). Gran Encuesta Integrada De Hogares (GEIH) 2017. Recuperado de: https:/www.dane.gov.co/index.php/estadisticas-por-tema/mercadolaboral/empleo-y-desempleo/geih-historicos 
Morales-González, Manjarrés de Ávila y Cruz-Almanza: Evaluación del Servicio Público...

Departamento Nacional de Planeación (2007). Programa Empleo en Acción: Impactos del programa sobre los beneficiarios y sus familias. Recupeardo de: http://www.dps.gov.co/ent/gen/prg/Documents/2007PROGRAMA \%20EMPLEO \%20EN \%20ACCION \%20IMPACTOS \%20SOBRE \%20LOS \%20JOVENES \%20Y\%20SUS $\%$ 20FAMILIAS.pdf

Departamento Nacional de Planeación (2008). Subprograma Jóvenes en Acción: Consultoría para evalaución de impacto del subprograma jóvenes en Acción. Evaluación de Politicas Públicas. Recuperado de: http://www.dps.gov.co/inf/doc/Jvenes \%20en \%20Accin/Informe $\% 20$ final \%20de \%20la \%20la \%20Evaluación \%20de \%20Impacto \%20del \%20Programa \%20Jóvenes \%20en \%20Acción-2017.pdf

Fedesarrollo (2009). Evaluación de Impacto de Tres Programas y Linea de Base de un Programa del Servicio Nacional de Aprendizaje - SENA. Recuperado de: https:/www.repository.fedesarrollo.org.co/bitstream/ handle/11445/345/Repor_Noviembre_2009_Santa $\% 20$ Maria_et_al.pdf?sequence $=1 \&$ isAllowed $=y$

Flores Lima, R. (2010). Innovaciones en la Evaluación de Impacto del Servicio de Intermediación Laboral en México (Notas Técnicas IDB-TN-118). Recuperado del sitio web del Banco Interamericano de Desarrollo: http://publications.iadb.org/handle/11319/2697

Heckman, J. J., Lalonde, R. J. \& Smith, J. A. (1999). The Economics and Econometrics of Active Labor Market Programs. En Handbook of Labor Economics (Vol. 3, Parte A) (pp. 1865-2097). Gran Bretaña: Elsevier BV.

Mincer, J. (1974). Schooling, Experience and Earnings. Recuperado de: https://www.nber.org/books/minc74-1

Observatorio del Serivicio Público de Empleo (2016a). Documento Técnico: Estrategias de intervención municipal del Servicio Público de Empleo 2016. Recuperado de http://serviciodeempleo.gov.co/ observatorio/2016/10/03/estrategias-municipales-spe-2016/ 
Observatorio del Servicio Público de Empleo (2016b). Coyuntura Laboral Caribe. Recuperado de: https://serviciodeempleo.gov.co/observatorio/wpcontent/uploads/dlm_uploads/2017/03/Bolet \%C3\%ADn-

Coyuntura-Labora-Caribe-1.pdf

Observatorio Regional del Mercado de Trabajo (ORMET Atlántico) (2017). Dinámica del mercado del trabajo del departamento del Atlántico y el Distrito de Barranquilla y su AM.

Prieto Bustos, O. (2009). Intermediación laboral. Semestre Económico, 12(23), 35-58.

Rojas Chavez, A. M. (2004). La intermediación laboral. Revista de Derecho, 22, 187-210. Recuperado de: http://rcientificas.uninorte.edu.co/ index.php/derecho/article/view/2847/1938

Uribe, J. I. \& Ortiz, C. H. (2006). Informalidad laboral en Colombia 19882000: Evolución, Teorias y Modelos. Cali: Universidad del Valle.

Uribe, J., Viáfara, C. \& Oviedo, Y. (2007). Efectividad de los canales de búsqueda de empleo en Colombia en el año 2003. Lecturas de Economía, $67,43-70$.

van Gameren, E. (2010). Evaluación de Impacto del Programa de Apoyo al Empleo. Recuperado de: http://guia.oitcinterfor.org/sites/default/files/ experiencias/Eval \%20impacto \%20prog \%20apoyo \%20empleo \%20Mx \%202008.pdf

Vera Rojas, C. P. (2005). Servicio Público de Empleo en el Perú: Teoría e impacto. Retrieved from http://www.academia.edu/4339144/ Servicio_Publico_de_Empleo_Teoria_e_Impacto 